Article

\title{
An Operator Based Approach to Irregular Frames of Translates
}

\author{
Peter Balazs ${ }^{1, *(1)}$ and Sigrid Heineken ${ }^{2}$ \\ 1 Acoustics Research Institute, Austrian Academy of Sciences, Wohllebengasse 12-14, 1040 Wien, Austria \\ 2 IMAS UBA-CONICET, Departamento de Matemática, Facultad de Ciencias Exactas y Naturales, \\ Universidad de Buenos Aires, Pabellón I, Ciudad Universitaria, C1428EGA Buenos Aires, Argentina; \\ sheinek@dm.uba.ar \\ * Correspondence: peter.balazs@oeaw.ac.at; Tel.: +43-1-51581-2510
}

Received: 29 March 2019; Accepted: 13 May 2019; Published: 20 May 2019

\begin{abstract}
We consider translates of functions in $L^{2}\left(\mathbb{R}^{d}\right)$ along an irregular set of points, that is, $\left\{\phi\left(\cdot-\lambda_{k}\right)\right\}_{k \in \mathbb{Z}}$-where $\phi$ is a bandlimited function. Introducing a notion of pseudo-Gramian function for the irregular case, we obtain conditions for a family of irregular translates to be a Bessel, frame or Riesz sequence. We show the connection of the frame-related operators of the translates to the operators of exponentials. This is used, in particular, to find for the first time in the irregular case a representation of the canonical dual as well as of the equivalent Parseval frame-in terms of its Fourier transform.
\end{abstract}

Keywords: frames; Riesz bases; irregular translates; canonical duals; frame-related operators.

\section{Introduction}

Frames of translates, i.e., frame sequences originated by the shifts of a given, fixed function, are an important mathematical background for Gabor [1-3], wavelet [4,5] and sampling theory [6,7]. One generating function $\phi$ is shifted to create the analyzing family of elements, $\left\{\phi\left(\cdot-\lambda_{k}\right)\right\}_{k \in \mathbb{Z}}$ for a sequence $\Lambda=\left\{\lambda_{k}\right\}_{k \in \mathbb{Z}}$. These systems play a main role in the theory of shift invariant spaces (SIS) [8-11], which is very useful for the modeling of problems in signal processing, and is central in approximation. It is also a very active field of research in mathematics, see e.g., [12-15]. Frames of translates are closely related to frames of exponentials also called Fourier frames [16-18].

The regular shifts, e.g., when $\lambda_{k}=k b$ for some fixed $b>0$, were studied e.g., in [19]. Investigations of irregular frames of translates, where the set $\Lambda$ has no regular structure, were done e.g., in $[1,6]$. But there remain several open questions, in particular how the concept of Gramian function could be defined in the irregular case, which is a fundamental tool for describing properties of regular systems of translates. Also no explicit formulas for the canonical dual of irregular frames of translates were given so far. We do this in the present paper.

The paper is structured as follows: In Section 2 we present an overview over the main results. In Section 3 we summarize basic notations and preliminaries. In Section 4 we generalize the concept of the Gramian function and use it to describe Bessel frame and Riesz properties of irregular shifts. In Section 5 we look at the inter-relation of the frame-related operators for those sequences to those of exponential functions. This permits us to study Bessel, frame and Riesz properties of irregular frames of translates, and furthermore give formulas for the canonical dual, and for equivalent Parseval frames.

\section{Main Results}

We state here our main results at the beginning of the paper. For notations and definitions pleaser refer to the later sections. 
For regular frames of translates the Gramian function is a key concept. For the case of irregular frames of translates, where we have no group structure, we introduce the following notion.

Definition 1. Let $\phi \in L^{2}\left(\mathbb{R}^{d}\right)$. We define its pseudo-Gramian function by

$$
\Phi=|\hat{\phi}|^{2}
$$

Surprisingly, as we will see in Sections 4 and 5, this definition leads to quite analogous results as in the regular case, where this function is used only in a periodized version. Among them we show the following:

Theorem 1. Let $\phi \in L^{2}\left(\mathbb{R}^{d}\right)$ such that $\sup p(\hat{\phi})$ is compact. In each of the following cases (i.e., (1) or (2)), if one of the following conditions (i.e., $(a),(b)$ or $(c))$ is fulfilled, the other two are equivalent:

(1) (a) $\quad\left\{e_{\lambda_{k}}\right\}_{k \in \mathbb{Z}}$ is a frame in $L^{2}(\operatorname{supp}(\hat{\phi}))$.

(b) $\left\{T_{\lambda_{k}} \phi\right\}_{k \in \mathbb{Z}}$ is a frame in $\operatorname{PW} W_{\text {supp }}(\hat{\phi})$.

(c) There exist $p, P>0$ such that $p \leq \Phi \leq P$ a.e. on $\operatorname{supp}(\hat{\phi})$.

(2) (a) $\quad\left\{e_{\lambda_{k}}\right\}_{k \in \mathbb{Z}}$ is a Riesz basis in $L^{2}(\operatorname{supp}(\hat{\phi}))$.

(b) $\quad\left\{T_{\lambda_{k}} \phi\right\}_{k \in \mathbb{Z}}$ is a Riesz basis in $P W_{\text {supp }(\hat{\phi})}$.

(c) There exist $p, P>0$ such that $p \leq \Phi \leq P$ a.e. on $\operatorname{supp}(\hat{\phi})$.

Note that the condition $1(c)=2(c)$ implies that the frame properties of $\left\{e_{\lambda_{k}}\right\}_{k \in \mathbb{Z}}$ and $\left\{T_{\lambda_{k}} \phi\right\}_{k \in \mathbb{Z}}$ coincide.

Theorem 1 is an extension of results in [20]. We prove it using an operator theory based approach. Furthermore we can give an explicit form for the canonical dual of an irregular set of translates, as a nice generalization of results in the regular case:

Theorem 2. Let $\phi \in L^{2}\left(\mathbb{R}^{d}\right)$ such that $\sup p(\hat{\phi})$ is compact. Assume that there exist $p, P>0$ such that $p \leq \Phi \leq P$ a.e. on $\sup p(\hat{\phi})$ and let $\left\{e_{\lambda_{k}}\right\}_{k \in \mathbb{Z}}$ be a frame sequence in $L^{2}(\sup p(\hat{\phi}))$. Then the canonical dual of $\left\{T_{\lambda_{k}} \phi\right\}_{k \in \mathbb{Z}}$ is $\left\{\theta_{k}\right\}_{k \in \mathbb{Z}}$ where

$$
\hat{\theta}_{k}=\left\{\begin{array}{cc}
\hat{\phi} \widetilde{\Phi} \widetilde{\lambda_{\lambda_{k}}} & \text { on } \operatorname{supp}(\hat{\phi}) \\
0 & \text { otherwise. }
\end{array}\right.
$$

In particular if $\left\{e_{\lambda_{k}}\right\}_{k \in \mathbb{Z}}$ is an A-tight frame, then $\theta_{k}=T_{\lambda_{k}} \theta$ with $\hat{\theta}=\frac{1}{A} \frac{1}{\hat{\phi}}(\operatorname{on} \operatorname{supp}(\hat{\phi}))$.

Note that the definition of the pseudo-Gramian gives a convenient way of formulating the other results, although they do not depend on this definition per-se.

\section{Notation and Preliminaries}

Given $\lambda \in \mathbb{R}^{d}$, we denote by $e_{\lambda}$ the function defined by $e_{\lambda}(x)=e^{-2 \pi i \lambda x}$. The operators $T_{\lambda}$ and $\mathcal{M}_{\lambda}$ are given by $T_{\lambda} f(x)=f(x-\lambda)$ and $\mathcal{M}_{\lambda} f(x)=e_{-\lambda} f(x)$ respectively. We write $\hat{f}$ for the Fourier transform, $\mathcal{F}: L^{2}\left(\mathbb{R}^{d}\right) \rightarrow L^{2}\left(\mathbb{R}^{d}\right)$, given by $\mathcal{F}(f)(w)=\hat{f}(w)=\int_{\mathbb{R}^{d}} f(x) e^{-2 \pi i x w} d x$, for $f \in L^{1}\left(\mathbb{R}^{d}\right) \cap L^{2}\left(\mathbb{R}^{d}\right)$ with the natural extension to $L^{2}\left(\mathbb{R}^{d}\right)$.

In this paper $E \subseteq \mathbb{R}^{d}$ is a bounded set. We denote

$$
P W_{E}=\left\{f \in L^{2}\left(\mathbb{R}^{d}\right) \mid \operatorname{supp}(\hat{f}) \subseteq E\right\}
$$

The space

$$
\left\{f \in L^{2}\left(\mathbb{R}^{d}\right): f(x)=0 \text { for a.e. } x \in \mathbb{R}^{d} \backslash E\right\}
$$


is a closed subspace of $L^{2}\left(\mathbb{R}^{d}\right)$ which is isomorphic to $L^{2}(E)$, and we will identify these two spaces. The Fourier transform maps $P W_{E}$ onto $L^{2}(E)$.

When we write that $\left\{e_{\lambda_{k}}\right\}_{k \in \mathbb{Z}}$ is a frame (frame sequence, Bessel sequence or Riesz basis) of $L^{2}(E)$ we will mean that the set $\left\{e_{\lambda_{k}} \chi_{E}\right\}_{k \in \mathbb{Z}}$ has that property, where $\chi_{E}$ stands for the indicator function of $E$, that is, it is an outer frame, Bessel or Riesz basis [21]. For a given operator $O$ we denote its pseudo-inverse by $O^{\dagger}$. Motivated by that, for a function $\psi$ we denote

$$
\psi^{\dagger}(x)=\left\{\begin{array}{cc}
1 / \psi(x) & x \in \operatorname{supp}(\psi) \\
0 & \text { otherwise }
\end{array}\right.
$$
by $R_{f}$.

Throughout this work $\Lambda=\left\{\lambda_{k}\right\}_{k \in \mathbb{Z}}$ will be a sequence in $\mathbb{R}^{d}$. For a function $f$ we denote its range

\subsection{Frames}

Let $\mathcal{H}$ be a separable Hilbert space. A sequence $\left\{\psi_{k}\right\}_{k \in \mathbb{Z}} \subseteq \mathcal{H}$ is a frame for $\mathcal{H}$ if there exist positive constants $A$ and $B$ that satisfy

$$
A\|f\|^{2} \leq \sum_{k \in \mathbb{Z}}\left|\left\langle f, \psi_{k}\right\rangle\right|^{2} \leq B\|f\|^{2} \quad \forall f \in \mathcal{H} .
$$

We denote the optimal bounds by $A^{(\text {opt })}$ and $B^{(o p t)}$. If $A^{(o p t)}=B^{(o p t)}$ then it is called a tight frame, and if $A^{(o p t)}=B^{(o p t)}=1$ a Parseval frame. If $\left\{\psi_{k}\right\}_{k \in \mathbb{Z}}$ satisfies the right inequality in the above formula, it is called a Bessel sequence.

A sequence $\left\{\psi_{k}\right\}_{k \in \mathbb{Z}} \subseteq \mathcal{H}$ is a Riesz basis for $\mathcal{H}$ if it is complete in $\mathcal{H}$ and there exist $0<A \leq B$ such that for every finite scalar sequence $\left\{c_{k}\right\}_{k \in \mathbb{Z}}$

$$
A \sum\left|c_{k}\right|^{2} \leq\left\|\sum_{k \in \mathbb{Z}} c_{k} \psi_{k}\right\|^{2} \leq B \sum\left|c_{k}\right|^{2} .
$$

The constants $A$ and $B$ are called Riesz bounds. A complete sequence is exact if it ceases to be complete when an arbitrary element is removed. Exact frames are precisely the Riesz bases.

We say that $\left\{\psi_{k}\right\}_{k \in \mathbb{Z}} \subseteq \mathcal{H}$ is a frame sequence (Riesz sequence) if it is a frame (Riesz basis) for its span.

For $\Psi=\left\{\psi_{k}\right\}_{k \in \mathbb{Z}}$ a Bessel sequence, its analysis operator $C: \mathcal{H} \rightarrow \ell^{2}(\mathbb{Z})$ is defined by $C(f)=$ $\left\{\left\langle f, \psi_{k}\right\rangle_{\mathcal{H}}\right\}_{k \in \mathbb{Z}}$, and its synthesis operator $D: \ell^{2}(\mathbb{Z}) \rightarrow \mathcal{H}$ by $D c=\sum_{k \in \mathbb{Z}} c_{k} \psi_{k}$.

If $\Psi$ is a frame of $\mathcal{H}$, the frame operator $S: \mathcal{H} \longrightarrow \mathcal{H}$ given by $S(f)=D C(f)=\sum_{k \in \mathbb{Z}}\left\langle f, \psi_{k}\right\rangle \psi_{k}$ is bounded, invertible, self-adjoint and positive. There always exists a dual frame of $\Psi$, which is a frame $\left\{\phi_{k}\right\}_{k \in \mathbb{Z}}$ such that

$$
f=\sum_{k \in \mathbb{Z}}\left\langle f, \phi_{k}\right\rangle \psi_{k} \forall f \in \mathcal{H} \quad \text { or } \quad f=\sum_{k \in \mathbb{Z}}\left\langle f, \psi_{k}\right\rangle \phi_{k} \forall f \in \mathcal{H}
$$

The sequence $\left\{S^{-1} \psi_{k}\right\}_{k \in \mathbb{Z}}$ satisfies (1). It is called the canonical dual frame of $\left\{\psi_{k}\right\}_{k \in \mathbb{Z}}$ and we will denote it by $\left\{\widetilde{\psi_{k}}\right\}_{k \in \mathbb{Z}}$. We distinguish between the operators by subscripts, e.g., $C_{e}$ is the analysis operator for the set of exponentials $\left\{e_{\lambda_{k}}\right\}_{k \in \mathbb{Z}}$ and $C_{\phi}$ those of the system of translates $\left\{T_{\lambda_{k}} \phi\right\}_{k \in \mathbb{Z}}$. For the operators corresponding to the canonical dual we will write $\tilde{C}_{e}$ respectively $\tilde{C}_{\phi}$.

To every frame $\Psi=\left\{\psi_{k}\right\}_{k \in \mathbb{Z}}$ a canonical tight frame can be associated, which is the sequence $\left\{S^{-\frac{1}{2}} \psi_{k}\right\}_{k \in \mathbb{Z}}$, where $S^{-\frac{1}{2}}$ is the positive square root of $S^{-1}$. 


\section{Gram Matrices}

The (bi-infinite) Gram matrix $G$ for $\Psi$ is given by $[G]_{j, m}=\left\langle\psi_{m}, \psi_{j}\right\rangle$, for $j, m \in \mathbb{Z}$. This matrix defines an operator by the standard matrix multiplication on $\ell^{2}(\mathbb{Z})$, the set of square summable sequences. It is known, that many properties of the sequence are directly related to properties of this operator, for a summary see (Prop. 4.4, [22]). For example, that $G$ is bounded, if and only if the sequence forms a Bessel sequence. $G$ is invertible on all of $\ell^{2}(\mathbb{Z})$ if and only if the sequence is a Riesz sequence.

\subsection{Multipliers}

In this work the operator that consists of a multiplication by a given function will play an important role.

For $L^{2}\left(\mathbb{R}^{d}\right)$ we can give the following results, which are either known, see for example, Reference [23] or can be easily proved:

Proposition 1. Let $\phi$ be a measurable function defined in $\mathbb{R}^{d}$, and consider the multiplication operator $M_{\phi}$ on $L^{2}\left(\mathbb{R}^{d}\right)$ given by $\left(M_{\phi}(f)\right)(t)=\phi(t) f(t)$.

1. $\phi \in L^{\infty}\left(\mathbb{R}^{d}\right)$ if and only if $M_{\phi}: L^{2}\left(\mathbb{R}^{d}\right) \rightarrow L^{2}\left(\mathbb{R}^{d}\right)$ is well defined and bounded. In this case $\|\phi\|_{\infty}=\left\|M_{\phi}\right\|_{O p}$.

2. Assume there exist $p, P>0$ such that $p \leq|\phi(x)| \leq P$ for almost all $x \in \operatorname{supp}(\phi)$. Then $\operatorname{Im}\left(M_{\phi}\right)=$ $L^{2}(\operatorname{supp}(\phi)), \operatorname{ker}\left(M_{\phi}\right)=L^{2}\left(\mathbb{R}^{d} \backslash \operatorname{supp}(\phi)\right)$ and $M_{\phi}^{+}=M_{\phi^{+}}$. On supp $(\phi)$ we have

$$
\left\|M_{\phi}(f)\right\| \geq p\|f\| .
$$

3. $M_{\phi}$ is boundedly invertible if and only if there exists $p>0$ such that $p \leq|\phi(x)|$ a.e. In this case $M_{\phi}^{-1}=M_{\frac{1}{\phi}}$.

\section{Gramian Function for Irregular Frames of Translates}

In this section we will show some results about sufficient and necessary conditions on the pseudo-Gramian function for Bessel sequence and Riesz basis properties.

As a connection of the pseudo-Gramian function with the Gramian matrix we get the following result.

Proposition 2. Let $\phi \in L^{2}\left(\mathbb{R}^{d}\right)$. For any system of translates $\left\{T_{\lambda_{k}} \phi\right\}_{k \in \mathbb{Z}}$ the Gramian matrix $G$ has the entries

$$
G_{k, l}=\hat{\Phi}\left(\lambda_{k}-\lambda_{l}\right) \text {. }
$$

Proof.

$$
\begin{gathered}
G_{k, l}=\left\langle T_{\lambda_{k}} \phi, T_{\lambda_{l}} \phi\right\rangle=\left\langle\phi, T_{\lambda_{l}-\lambda_{k}} \phi\right\rangle= \\
=\left\langle\hat{\phi}, M_{\lambda_{k}-\lambda_{l}} \hat{\phi}\right\rangle=\int_{\mathbb{R}^{d}}|\hat{\phi}(\xi)|^{2} e^{-2 \pi i\left(\lambda_{k}-\lambda_{l}\right) \xi} d \xi=\left(\mathcal{F}|\hat{\phi}|^{2}\right)\left(\lambda_{k}-\lambda_{l}\right)
\end{gathered}
$$

As a consequence of Schur's test, see for example, Reference [2] and the properties of the Gram matrix we get:

Corollary 1. Let $\phi \in L^{2}\left(\mathbb{R}^{d}\right)$. If

$$
\sup _{k \in \mathbb{Z}} \sum_{l \in \mathbb{Z}}\left|\hat{\Phi}\left(\lambda_{k}-\lambda_{l}\right)\right|<\infty,
$$

then the system of translates $\left\{T_{\lambda_{k}} \phi\right\}_{k \in \mathbb{Z}}$ is a Bessel sequence. 
This is equivalent to the Gram matrix being included in the Schur-type matrix algebra with no weight, that is, the set of translates forms an intrinsically localized frame [24,25].

On the other hand using necessary conditions for bounded matrices on $\ell^{2}[26,27]$ the following can be shown:

Corollary 2. If $\phi \in L^{2}\left(\mathbb{R}^{d}\right)$ and $\left\{T_{\lambda_{k}} \phi\right\}_{k \in \mathbb{Z}}$ is a Bessel sequence, then

$$
\sup _{k \in \mathbb{Z}} \sum_{l \in \mathbb{Z}}\left|\hat{\Phi}\left(\lambda_{k}-\lambda_{l}\right)\right|^{2}<\infty
$$

For Riesz bases we obtain the following result:

Proposition 3. Let $\phi \in L^{2}\left(\mathbb{R}^{d}\right)$. If

$$
\|\phi\|_{L^{2}\left(\mathbb{R}^{d}\right)}^{2}>\sup _{j \in \mathbb{Z}}\left(\sum_{l, l \neq j}\left|\hat{\Phi}\left(\lambda_{j}-\lambda_{l}\right)\right|\right),
$$

then the system of translates $\left\{T_{\lambda_{k}} \phi\right\}_{k \in \mathbb{Z}}$ is a Riesz sequence.

Proof. As $\phi \in L^{2}\left(\mathbb{R}^{d}\right)$ we have

$$
\hat{\Phi}(0)=\int_{\mathbb{R}^{d}} \Phi(\omega) d \omega=\int_{\mathbb{R}^{d}}|\hat{\phi}(\omega)|^{2} d \omega=\|\hat{\phi}\|^{2}=\|\phi\|^{2} .
$$

Therefore, by assumption, the Gram matrix $G_{k, l}=\hat{\Phi}\left(\lambda_{k}-\lambda_{l}\right)$ is strictly diagonally dominant, and it is invertible by (Lemma 6.5.4, [2]), which is the infinite-dimensional version of the Levy-Desplanque theorem. The Gram-matrix is therefore invertible from $\ell^{2}$ onto $\ell^{2}$ and so the sequence is a Riesz sequence [1].

\section{The Operator-Based Approach to Irregular Frames of Translates}

\subsection{Irregular Translates in $P W_{E}$}

From now on we will assume $\phi \in P W_{E}$. Analogous as in (Theorem 4.1, Prop. 3.6, [20]) the following can be proved.

\section{Lemma 1.}

1. Assume $\left\{e_{\lambda_{k}}\right\}_{k \in \mathbb{Z}}$ is a Bessel sequence of $L^{2}(E)$ with bound $B_{e}$ and there exists $P>0$ such that $\Phi \leq P$ a.e. Then $\left\{T_{\lambda_{k}} \phi\right\}_{k \in \mathbb{Z}}$ is a Bessel sequence for $L^{2}\left(\mathbb{R}^{d}\right)$ with bound $B_{\phi}=B_{e} \cdot P$.

2. Let $\left\{T_{\lambda_{k}} \phi\right\}_{k \in \mathbb{Z}}$ be a Bessel sequence for $L^{2}\left(\mathbb{R}^{d}\right)$ with bound $B_{\phi}$, and assume there exists $p>0$ such that $\Phi \geq p$ a.e. in $E$. Then $\left\{e_{\lambda_{k}}\right\}_{k \in \mathbb{Z}}$ is a Bessel sequence for $L^{2}(E)$ with bound $B_{e}=B_{\phi} / p$.

The following results generalize Lemmas 7.2.1 and 7.3.2 in [1].

Lemma 2. Let $\left\{e_{\lambda_{k}}\right\}_{k \in \mathbb{Z}}$ be a Bessel sequence for $L^{2}(E)$ and assume there exists $P>0$ such that $\Phi(\omega) \leq P$ a.e. Let $\left\{c_{k}\right\}_{k \in \mathbb{Z}} \in \ell^{2}(\mathbb{Z})$. Then $\sum_{k \in \mathbb{Z}} c_{k} T_{\lambda_{k}}$ क converges unconditionally in $L^{2}\left(\mathbb{R}^{d}\right), \sum_{k \in \mathbb{Z}} c_{k} e_{\lambda_{k}}$ converges unconditionally in $L^{2}(E)$ and

$$
\mathcal{F}\left(\sum_{k \in \mathbb{Z}} c_{k} T_{\lambda_{k}} \phi\right)=\left(\sum_{k \in \mathbb{Z}} c_{k} e_{\lambda_{k}}\right) \hat{\phi} .
$$

Therefore $R_{M_{\hat{\phi}_{\mid R_{D_{e}}}}}=\mathcal{F}\left(R_{D_{\phi}}\right)$ and $f \in R_{D_{\phi}}$ if and only if there exists $F \in R_{D_{e}}$ such that $\hat{f}=F \cdot \hat{\phi}$. 
Proof. Following Lemma $1\left\{T_{\lambda_{k}} \phi\right\}_{k \in \mathbb{Z}}$ is a Bessel sequence and so all involved sums converge unconditionally.

$$
\mathcal{F}\left(\sum_{k \in \mathbb{Z}} c_{k} T_{\lambda_{k}} \phi\right)=\sum_{k \in \mathbb{Z}} c_{k} e_{\lambda_{k}} \hat{\phi} .
$$

As $\hat{\phi}$ is bounded, the multiplication operator $M_{\hat{\phi}}$ is also bounded and therefore

$$
\left(\sum_{k \in \mathbb{Z}} c_{k} e_{\lambda_{k}}\right) \hat{\phi}=M_{\hat{\phi}}\left(\sum_{k \in \mathbb{Z}} c_{k} e_{\lambda_{k}}\right)=\sum_{k \in \mathbb{Z}} c_{k} M_{\hat{\phi}}\left(e_{\lambda_{k}}\right)=\sum_{k \in \mathbb{Z}} c_{k} e_{\lambda_{k}} \hat{\phi}
$$

Corollary 3. Let $\left\{e_{\lambda_{k}}\right\}_{k \in \mathbb{Z}}$ be a Bessel sequence in $L^{2}(E)$ and assume there exists $P>0$ such that $\Phi \leq P$ a.e. Let $\left\{T_{\lambda_{k}} \phi\right\}_{k \in \mathbb{Z}}$ be a frame sequence. Then $R_{D_{e}} \cdot \hat{\phi}=\overline{\operatorname{span}}\left\{e_{\lambda_{k}} \hat{\phi}: k \in \mathbb{Z}\right\}=\mathcal{F}\left(\overline{\operatorname{span}}\left\{T_{\lambda_{k}} \phi: k \in \mathbb{Z}\right\}\right)$ and so $f \in \overline{\operatorname{span}}\left\{T_{\lambda_{k}} \phi: k \in \mathbb{Z}\right\}$ if and only if there exists $F \in R_{D_{e}}$ such that $\hat{f}=F \cdot \hat{\phi}$.

Proof. In this case $\overline{\operatorname{span}}\left\{T_{\lambda_{k}} \phi: k \in \mathbb{Z}\right\}=R_{D_{\phi}}$. We see from the proof of Lemma 2 that $F=\sum_{k \in \mathbb{Z}}\left\langle f, \widetilde{T_{\lambda_{k}} \phi}\right\rangle e_{\lambda_{k}}$.

\subsection{Relation of Operators}

In this section we state the relations of the operators of the set of exponentials and the system of translates.

Lemma 3. Let $\left\{e_{\lambda_{k}}\right\}_{k \in \mathbb{Z}}$ be a Bessel sequence of $L^{2}(E)$ and assume that there exists $P>0$ such that $\Phi \leq P$ a.e. Then $\left\{T_{\lambda_{k}} \phi\right\}$ is a Bessel sequence of $L^{2}\left(\mathbb{R}^{d}\right)$ and

$$
\begin{array}{ll}
D_{\phi}=\mathcal{F}^{-1} M_{\hat{\phi}} D_{e}, & C_{\phi}=C_{e} M_{\bar{\phi}} \mathcal{F}, \\
S_{\phi}=\mathcal{F}^{-1} M_{\hat{\phi}} S_{e} M_{\overline{\hat{\phi}}} \mathcal{F} & G_{\phi}=C_{e} M_{\Phi} D_{e} .
\end{array}
$$

Proof. By Lemma $1\left\{T_{\lambda_{k}} \phi\right\}_{k \in \mathbb{Z}}$ is a Bessel sequence of $L^{2}\left(\mathbb{R}^{d}\right)$. For $c \in \ell^{2}(\mathbb{Z})$, by Lemma 2,

$$
\mathcal{F}\left(D_{\phi} c\right)=\mathcal{F}\left(\sum_{k} c_{k} T_{\lambda_{k}} \phi\right)=\left(\sum_{k} c_{k} e_{\lambda_{k}}\right) \hat{\phi}=M_{\hat{\phi}} D_{e} c .
$$

For $f \in P W_{E}$

$$
C_{\phi} f=\left\{\left\langle f, T_{\lambda_{k}} \phi\right\rangle\right\}_{k \in \mathbb{Z}}=\left\{\left\langle\hat{f}, e_{\lambda_{k}} \hat{\phi}\right\rangle\right\}_{k \in \mathbb{Z}}=\left\{\left\langle\hat{f} \bar{\phi}, e_{\lambda_{k}}\right\rangle\right\}_{k \in \mathbb{Z}} .
$$

So

$$
S_{\phi}=D_{\phi} C_{\phi}=\mathcal{F}^{-1} M_{\hat{\phi}} D_{e} C_{e} M_{\bar{\phi}} \mathcal{F}=\mathcal{F}^{-1} M_{\hat{\phi}} S_{e} M_{\bar{\phi}} \mathcal{F}
$$

and

$$
G_{\phi}=C_{\phi} D_{\phi}=C_{e} M_{\bar{\phi}} \mathcal{F} \mathcal{F}^{-1} M_{\hat{\phi}} D_{e}=C_{e} M_{|\hat{\phi}|^{2}} D_{e}
$$

In particular we have that $R_{D_{\phi}} \subseteq \mathcal{F}^{-1} L^{2}(\operatorname{supp}(\hat{\phi}))$. 
Proposition 4. Let $\left\{e_{\lambda_{k}}\right\}_{k \in \mathbb{Z}}$ be a frame of $L^{2}(\operatorname{supp}(\hat{\phi}))$, and assume there exist $p, P>0$ such that $p \leq \Phi \leq P$ a.e. on $\operatorname{supp}(\hat{\phi})$. Then $\left\{T_{\lambda_{k}} \phi\right\}_{k \in \mathbb{Z}}$ is a frame sequence that spans $P W_{\text {supp }(\hat{\phi})}$. Furthermore

$$
\begin{aligned}
& S_{\phi}^{-1}{ }_{\mid P W_{\text {supp }(\hat{\phi})}}=\mathcal{F}^{-1}\left(M_{\frac{1}{\bar{\phi}}} S_{e}^{-1} M_{\frac{1}{\phi}}\right) \mathcal{F}, \quad S_{\phi}^{\dagger}=\mathcal{F}^{-1} M_{\frac{1}{\bar{\phi}}} S_{e}^{-1} M_{\hat{\phi}^{+}} \mathcal{F}, \\
& D_{e}=M_{\bar{\phi}} \mathcal{F} D_{\phi}, \quad C_{e}=C_{\phi} \mathcal{F}^{-1} M_{\hat{\phi}^{\dagger}}{ }^{\prime} \\
& S_{e}=M_{\frac{1}{\phi}} \mathcal{F} S_{\phi} \mathcal{F}^{-1} M_{\bar{\phi}^{\dagger}}, \quad G_{e}=C_{\phi} \mathcal{F} M_{\frac{1}{\Phi}} \mathcal{F}^{-1} D_{\phi} . \\
& \text { Moreover } p A_{e}^{(o p t)} \leq A_{\phi}^{(o p t)} \leq A_{e}^{(o p t)} P \text { and } p B_{e}^{(o p t)} \leq B_{\phi}^{(o p t)} \leq B_{e}^{(o p t)} P \text {. }
\end{aligned}
$$

Proof. By Proposition $1 M_{\hat{\phi}}$ is invertible on $L^{2}(\operatorname{supp}(\hat{\phi}))$, and therefore $S_{\phi}=\mathcal{F}^{-1} M_{\hat{\phi}} S_{e} M_{\hat{\phi}} \mathcal{F}$ is invertible on $P W_{\sup p(\hat{\phi})}$, and

$$
\left.S_{\phi}^{-1}\right|_{\mid P W_{\text {supp }(\hat{\phi})}}=\mathcal{F}^{-1} M_{\frac{\overline{1}}{\bar{\phi}}} S_{e}^{-1} M_{\overline{1}} \mathcal{F}
$$

Note that $\mathcal{F}$ maps $P W_{E}$ onto $L^{2}(E)$ and $M_{\hat{\phi}}$ maps $L^{2}(E)$ onto $L^{2}(\operatorname{supp}(\hat{\phi}))$, with $M_{\hat{\phi}}^{+}=M_{\hat{\phi}^{+}}$. Therefore $S_{\phi}^{\dagger}=\mathcal{F}^{-1} M_{\frac{1}{\bar{\phi}}} S_{e}^{-1} M_{\hat{\phi}^{+}} \mathcal{F}$.

By Lemma 2, $R_{D_{\phi}} \subseteq P W_{\operatorname{supp}(\hat{\phi})}$ and since $\left\{e_{\lambda_{k}}\right\}_{k \in \mathbb{Z}}$ is a frame for $L^{2}(\operatorname{supp}(\hat{\phi}))$, we know that $R_{D_{\phi}}=P W_{\text {supp }(\hat{\phi})}$. Now $D_{\phi}=\mathcal{F}^{-1} M_{\hat{\phi}} D_{e}$ implies $M_{\hat{\phi}}^{-1} \mathcal{F} D_{\phi}=D_{e}$, and so $R_{D_{e}}=L^{2}(\operatorname{supp}(\hat{\phi}))$.

We have $C_{\phi} \mathcal{F}^{-1}=C_{e} M_{\bar{\phi}}$ and hence $C_{e}=C_{\phi} \mathcal{F}^{-1} M_{\bar{\phi}^{+}}$.

So

$$
G_{e}=C_{e} D_{e}=C_{\phi} \mathcal{F}^{-1} M_{\hat{\phi}^{\dagger}} M_{\hat{\phi}} \mathcal{F} D_{\phi}=C_{\phi} \mathcal{F}^{-1} M_{\frac{1}{|\hat{\phi}|^{2}}} \mathcal{F} D_{\phi}
$$

Finally,

$$
\frac{1}{A_{\phi}^{(o p t)}}=\left\|S_{\phi}^{-1}\right\|_{O p}=\left\|\mathcal{F}^{-1} M_{\frac{1}{\bar{\phi}}} S_{e}^{-1} M_{\frac{1}{\hat{\phi}}} \mathcal{F}\right\|_{O p} \leq \frac{1}{p} \frac{1}{A_{e}^{(o p t)}}
$$

and

$$
\frac{1}{A_{e}^{(o p t)}}=\left\|S_{e}^{-1}\right\|_{O p}=\left\|M_{\bar{\phi}} \mathcal{F} S_{\phi}^{-1} \mathcal{F}^{-1} M_{\hat{\phi}}\right\|_{O p} \leq P \frac{1}{A_{\phi}^{(o p t)}}
$$

Analogously, since $S_{\phi}=\mathcal{F}^{-1} M_{\hat{\phi}} S_{e} M_{\bar{\phi}} \mathcal{F}$, it can be proved that

$$
p B_{e}^{(o p t)} \leq B_{\phi}^{(o p t)} \leq B_{e}^{(o p t)} P .
$$

Furthermore, we can state the following result about the exactness of sequences of translates and sequences of exponentials. Observe that Proposition 2 follows also from the equation $G_{\phi}=C_{\phi} D_{\phi}=$ $C_{e} M_{\Phi} D_{e}$ in Lemma 3.

Corollary 4. Let $\left\{e_{\lambda_{k}}\right\}_{k \in \mathbb{Z}}$ be a Bessel sequence of $L^{2}(E)$.

1. Assume there exists $P>0$ such that $\Phi \leq P$ a.e.. If $\left\{T_{\lambda_{k}} \phi\right\}_{k \in \mathbb{Z}}$ is exact, then $\left\{e_{\lambda_{k}}\right\}_{k \in \mathbb{Z}}$ is exact.

2. Assume there exist $p, P>0$ such that $p \leq \Phi \leq P$ a.e. in $E$. Then $\left\{T_{\lambda_{k}} \phi\right\}_{k \in \mathbb{Z}}$ is exact if and only if $\left\{e_{\lambda_{k}}\right\}_{k \in \mathbb{Z}}$ is exact.

Proof. Lemma 3 yields $k e r D_{e} \subseteq k e r D_{\phi}$.

If $|\hat{\phi}|$ is bounded from above and below, then $M_{\hat{\phi}}$ is invertible and so by Proposition 4, part 2 follows.

We can now proof one of the theorems stated in Section 2. 
Proof of Theorem 1. (1) Since $\hat{\phi}$ is bounded from above and from below, $M_{\hat{\phi}}$ is invertible and so by Lemma $3(c) \Longrightarrow((b) \Longleftrightarrow(a))$. If $D_{e}$ and $D_{\phi}$ are surjective as well as $C_{e}$ and $C_{\phi}$ are injective, $M_{\hat{\phi}}$ is bijective and so the other directions are shown.

(2) follows from Corollary 4 applied to $E=\operatorname{supp}(\hat{\phi})$ and (1).

Note that if the completeness assumption is dropped (i.e., we use frame sequences and Riesz sequence) we still have the following:

$$
(c) \Longrightarrow((a) \Longleftrightarrow(b))
$$

and therefore

$$
(a) \Longrightarrow((c) \Longrightarrow(b)) \quad(b) \Longrightarrow((c) \Longrightarrow(a)) .
$$

For upper semi-frames [28], that is, complete Bessel sequences, similar relations can be given but not equivalencies.

Using Theorem 1, we can generalize Proposition 7.3.6. in [1] in the following sense:

Corollary 5. Let $\left\{e_{\lambda_{k}}\right\}_{k \in \mathbb{Z}}$ be a frame in $L^{2}(\sup p(\hat{\phi}))$. If $T_{\lambda_{k}} \phi$ forms a frame, then $\hat{\phi}$ is discontinuous and so $\phi \notin L^{1}\left(\mathbb{R}^{d}\right)$.

\subsection{The Canonical Dual}

We arrive to the relation between the canonical duals of frame sequences of exponentials and frame sequences of translates stated in Theorem 2.

Proof of Theorem 2. As in Proposition 4

$$
S_{\phi}^{-1}=\mathcal{F}^{-1} M_{\overline{\underline{\phi}}} S_{e}^{-1} M_{\frac{1}{\bar{\phi}}} \mathcal{F}
$$

and therefore

$$
\begin{gathered}
S_{\phi}^{-1} T_{\lambda_{k}} \phi=\mathcal{F}^{-1} M_{\frac{\bar{\phi}}{\bar{\phi}}} S_{e}^{-1} M_{\frac{1}{\phi}} \mathcal{F} T_{\lambda_{k}} \phi=\mathcal{F}^{-1} M_{\frac{\overline{1}}{\bar{\phi}}} S_{e}^{-1} M_{\frac{1}{\phi}} e_{\lambda_{k}} \hat{\phi}= \\
=\mathcal{F}^{-1} M_{\overline{\bar{\phi}}} S_{e}^{-1} e_{\lambda_{k}}=\mathcal{F}^{-1} M_{\frac{1}{\bar{\phi}}} \widetilde{e_{\lambda_{k}}}=\mathcal{F}^{-1} M_{\frac{\hat{\phi}}{|\hat{\phi}|^{2}}} \widetilde{e_{\lambda_{k}}} .
\end{gathered}
$$

From Lemma 3 we obtain:

Corollary 6. Assume $\left\{e_{\lambda_{k}}\right\}_{k \in \mathbb{Z}}$ is an $A_{e}$-tight frame of $L^{2}(E)$, and that there exist $P>0$ such that $\Phi \leq P$ a.e. Then $\left\{T_{\lambda_{k}} \phi\right\}$ is a Bessel sequence of $L^{2}\left(\mathbb{R}^{d}\right)$ and the frame operator of $\left\{T_{\lambda_{k}} \phi\right\}_{k \in \mathbb{Z}}$ is

$$
S_{\phi}=\mathcal{F}^{-1} M_{A_{e} \cdot \Phi} \mathcal{F}
$$

The following Corollary shows the relation between the analysis and synthesis operator of the duals of the shifts and of the exponentials.

Corollary 7. Assume that there exist $p, P>0$ such that $p \leq \Phi \leq P$ a.e. on $\sup p(\hat{\phi})$ and let $\left\{e_{\lambda_{k}}\right\}_{k \in \mathbb{Z}}$ be a frame sequence in $L^{2}(\operatorname{supp}(\hat{\phi}))$. Then

$$
\begin{gathered}
C_{\theta}=\widetilde{C_{\phi}}=\widetilde{C_{e}} M_{\hat{\phi}} \mathcal{F} . \\
D_{\theta}=\widetilde{D_{\phi}}=\mathcal{F}^{-1} M_{\frac{\hat{\phi}}{\Phi}} \widetilde{D_{e}} .
\end{gathered}
$$

Now let us investigate the operator $S^{1 / 2}$. 


\section{Lemma 4.}

1. Let $\Phi=1$ a.e. Then $S_{\phi}^{-1 / 2}=\mathcal{F}^{-1} M_{\hat{\phi}} S_{e}^{-1 / 2} M_{\bar{\phi}} \mathcal{F}$.

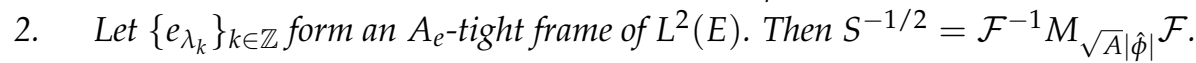

Proof. 1. With the assumption $M_{\hat{\phi}}$ is a unitary operator. Therefore

$$
\sqrt{S_{\phi}^{-1}}=\sqrt{\mathcal{F}^{-1} M_{\frac{1}{\bar{\phi}}} S_{e}^{-1} M_{\frac{1}{\hat{\phi}}} \mathcal{F}}=\mathcal{F}^{-1} M_{\hat{\phi}} S_{e}^{-1 / 2} M_{\bar{\phi}} \mathcal{F}
$$

2. By Corollary $6, S_{\phi}=\mathcal{F}^{-1} M_{A_{e}|\hat{\phi}|^{2}} \mathcal{F}$, and so $\sqrt{S_{\phi}}=\mathcal{F}^{-1} M_{\sqrt{A}|\hat{\phi}|} \mathcal{F}$.

Finally, we can give the following representation for an equivalent Parseval frame to a frame sequence of translates:

Theorem 3. Let $\left\{e_{\lambda_{k}}\right\}_{k \in \mathbb{Z}}$ be a frame for $L^{2}(\sup p(\hat{\phi}))$, and assume there exist $p, P>0$ such that $p \leq \Phi \leq P$ a.e. on $\operatorname{supp}(\hat{\phi})$. Set

$$
\hat{\theta_{k}^{\#}}=\left\{\begin{array}{cc}
\frac{\hat{\phi}}{\sqrt{\Phi}} e_{\lambda_{k}}^{\#} & \text { on } \operatorname{supp}(\Phi) \\
0 & \text { otherwise }
\end{array},\right.
$$

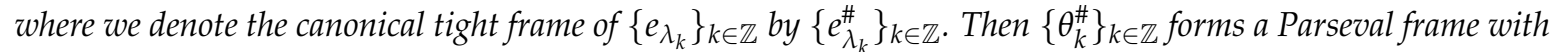

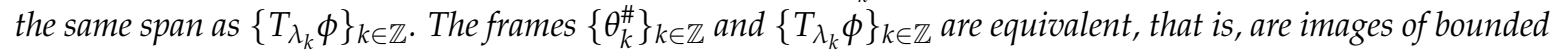
and boudedly invertible operator of each other.

If $\left\{e_{\lambda_{k}}\right\}_{k \in \mathbb{Z}}$ forms an $A_{e}$-tight frame, then $\left\{\theta_{k}^{\#}\right\}_{k \in \mathbb{Z}}$ forms a tight frame of translates with shifts $\lambda_{k}$ and generator $\frac{1}{A_{e}} \mathcal{F}^{-1} \frac{\hat{\phi}}{\sqrt{\Phi}}$.

Proof. By (Theorem 4.1, [20]) $\theta_{k}^{\#}$ is a frame sequence. Clearly $\overline{\operatorname{span}}\left\{\theta_{k}^{\#}: k \in \mathbb{Z}\right\} \subseteq \operatorname{PW}$ supp $(\hat{\phi})$. For $f \in P W_{\text {supp }}(\hat{\phi})$,

$$
\begin{gathered}
\sum_{k}\left\langle\hat{f}, \frac{\hat{\phi}}{\sqrt{\Phi}} e_{\lambda_{k}}^{\#}\right\rangle \frac{\hat{\phi}}{\sqrt{\Phi}} e_{\lambda_{k}}^{\#}=\left(\sum_{k}\left\langle\overline{\frac{\hat{\phi}}{\sqrt{\Phi}}} \hat{f}, e_{\lambda_{k}}^{\#}\right\rangle e_{\lambda_{k}}^{\#}\right) \cdot \frac{\hat{\phi}}{\sqrt{\Phi}}= \\
=\frac{\hat{\phi}}{\sqrt{\Phi}} \hat{f} \cdot \frac{\hat{\phi}}{\sqrt{\Phi}}=\hat{f} .
\end{gathered}
$$

Therefore $S=I d$ and $\overline{\operatorname{span}}\left\{\theta_{k}^{\#}: k \in \mathbb{Z}\right\}=P W_{\text {supp }(\hat{\phi})}$. The equivalence follows from the fact that by assumption $M_{\frac{1}{\sqrt{\Phi}}}$ is a bijection and so $R_{C_{\theta_{k}^{\#}}}=R_{C_{\phi}}$.

The result follows from Proposition 4.

\section{Outlook and Conclusions}

We have shown that under not-that-strong assumptions, we could, surprisingly, get similar results for the irregular setting as in the regular case, in particular for the (generalization of the) Gramian function and a formula for the canonical dual and Parseval frame.

We focused on the generalization of the sampling set to an irregular set. To handle the problem in full detail within the scope of this paper, we sticked to the bandlimitness assumption. Some of the results shown above are still valid for not band-limited functions, for example the results in Section 5.1 and Lemma 3. In the future an interesting question is what other results can be proved for this case.

We phrased the assumptions on the set of exponentials in a very general way, on purpose. In this way they can be easily combined with results about frames of exponentials and density results, see e.g., (Section 7.6, [1]). 
Author Contributions: Conceptualization, P.B. and S.H.; Formal analysis, P.B. and S.H.; Investigation, P.B. and S.H.; Methodology, P.B. and S.H.; Writing—original draft, P.B. and S.H.; Writing-review \& editing, P.B. and S.H.

Funding: This research was funded by the Austrian Science Fund (FWF) START-project FLAME (Frames and Linear Operators for Acoustical Modeling and Parameter Estimation; Y 551-N13), and the FP7 project PIEF-GA-2008-221090. We also thank MINCyT (Argentina) and BMWF-OeAD-WTZ (Austria) for the support of the bilateral project Shift Invariant Spaces And Its Applications To Audio Signal Processing; AU /10/25. Furthermore we acknowledge the partial support of PIP 112-201501-00589-CO (CONICET) and PROICO 03-1618 (UNSL).

Acknowledgments: The first author thanks Nora Simovich for help with typing. This publication is supported by the open access funding by the Austrian Science Fund (FWF).

Conflicts of Interest: The authors declare no conflict of interest.

\section{References}

1. Christensen, O. An Introduction to Frames and Riesz Bases; Birkhäuser: Boston, MA, USA, 2003.

2. Gröchenig, K. Foundations of Time-Frequency Analysis; Birkhäuser: Boston, MA, USA, 2001.

3. Janssen, A. Gabor representation of generalized functions. J. Math. Anal. Appl. 1981, 83, 377-394. [CrossRef]

4. Benedetto, J.J.; Treiber, O. Wavelet Transforms and Time-Frequency Signal Analysis. In Wavelet Frames: Multiresolution Analysis and Extension Principles; Birkhäuser: Boston, MA, USA, 2001.

5. Daubechies, I. Ten Lectures on Wavelets; SIAM: Philadelphia, PA, USA, 1992.

6. Aldroubi, A.; Gröchenig, K. Non-uniform sampling and reconstruction in shift-invariant spaces. SIAM Rev. 2001, 43, 585-620. [CrossRef]

7. Benedetto, J.J.; Ferreira, P. Modern Sampling Theory; Birkhäuser: Boston, MA, USA, 2004.

8. De Boor, C.; Devore, R.A.; Ron, A. Approximation from shift-invariant subspaces of $L^{2}\left(\mathbb{R}^{d}\right)$. Trans. Am. Math. Soc. 1994, 341, 787-806.

9. De Boor, C.; Devore, R.A.; Ron, A. The structure of finitely generated shift-invariant subspaces in $L^{2}\left(\mathbb{R}^{d}\right)$. J. Funct. Anal. 1994, 119, 37-78. [CrossRef]

10. Bownik, M. The structure of shift-invariant subspaces of $L^{2}\left(\mathbb{R}^{n}\right)$. J. Funct. Anal. 2000, 177, $282-309$. [CrossRef]

11. Koo, Y.; Lim, J. Perturbation of frame sequences and its applications to shift-invariant spaces. Linear Algebra Its Appl. 2007, 420, 295-309. [CrossRef]

12. Cabrelli, C.; Mosquera, C.; Paternostro, V. Linear combinations of frame generators in systems of translates. J. Math. Anal. Appl. 2014, 413, 776-788. [CrossRef]

13. Cabrelli, C.A.; Paternostro, V. Shift invariant spaces on LCA groups. J. Funct. Anal. 2010, 258, $2034-2059$. [CrossRef]

14. García, A.G.; Perez-Villalón, G. Multivariate generalized sampling in shift-invariant spaces and its approximation properties. J. Math. Anal. Appl. 2009, 355, 397-413. [CrossRef]

15. Tessera, R.; Wang, H. Uncertainty principles in finitely generated shift-invariant spaces with additional invariance. J. Math. Anal. Appl. 2014, 410, 134-143. [CrossRef]

16. Ortega Cerdà, J.; Seip, K. Fourier frames. Ann. Math. 2002, 155, 789-806. [CrossRef]

17. Duffin, R.J.; Schaeffer, A.C. A class of nonharmonic Fourier series. Trans. Am. Math. Soc. 1952, 72, $341-366$. [CrossRef]

18. Seip, K. On the connection between exponential bases and certain related sequences in $l^{2}(-\pi, \pi)$. J. Funct. Anal. 1995, 130, 131-160. [CrossRef]

19. Casazza, P.; Christensen, O.; Kalton, N.J. Frames of translates. Collect. Math. 2001, 1, 35-54.

20. Balazs, P.; Cabrelli, C.; Heineken, S.; Molter, U. Frames by multiplication. Curr. Dev. Theory Appl. Wavelets 2011, 5, 165-186.

21. Aldroubi, A.; Cabrelli, C.; Molter, U. Wavelets on irregular grids with arbitrary dilation matrices, and frame atoms for $L^{2}\left(R^{d}\right)$. Appl. Comput. Harmon. Anal. 2004, 17, 119-140. [CrossRef]

22. Balazs, P.; Stoeva, D.; Antoine, J.-P. Classification of general sequences by frame-related operators. Sampl. Theory Signal Process. 2011, 10, 151-170.

23. Conway, J.B. A Course in Functional Analysis, 2nd ed.; Graduate Texts in Mathematics; Springer: New York, NY, USA, 1990. 
24. Balazs, P.; Gröchenig, K. A Guide to Localized Frames and Applications to Galerkin-like Representations of Operators. In Frames and Other Bases in Abstract and Function Spaces; Pesenson, I., Mhaskar, H., Mayeli, A., Gia, Q.L., Zhou, D., Eds.; Birkhäuser: Boston, MA, USA; Springer: Berlin/Heidelberg, Germany, 2017.

25. Fornasier, M.; Gröchenig, K. Intrinsic localization of frames. Constr. Approx. 2005, 22, 395-415. [CrossRef]

26. Crone, L. A characterization of matrix operator on $l^{2}$. Math. Z. 1971, 123, 315-317. [CrossRef]

27. Maddox, I.J. Infinite Matrices of Operators; Lecture Notes in Mathematics; Springer: Berlin, Germany, 1980.

28. Antoine, J.-P.; Balazs, P. Frames and Semi-Frames. J. Phys. A Math. Theor. 2011, 44, 205201. [CrossRef]

(C) 2019 by the authors. Licensee MDPI, Basel, Switzerland. This article is an open access article distributed under the terms and conditions of the Creative Commons Attribution (CC BY) license (http:/ / creativecommons.org/licenses/by/4.0/). 